Journal of Applied Fluid Mechanics, Vol. 9, Special Issue 1, pp. 107-111, 2016.

Selected papers from the $7^{\text {th }}$ International Exergy, Energy

and Environment Symposium, IEEE7-2015

Available online at www.jafmonline.net, ISSN 1735-3572, EISSN 1735-3645.

DOI: $10.36884 /$ jafm.9.SI1.25820

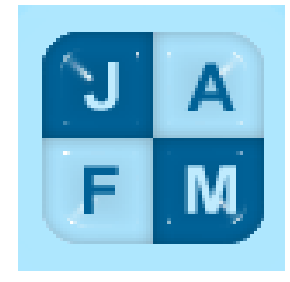

\title{
Aerodynamic Investigation of a Wind Turbine using CFD and Modified BEM Methods
}

\author{
M. Tahani ${ }^{\dagger}$, M. Moradi \\ ${ }^{1}$ University of Tehran, Faculty of New Sciences and Technologies, End of North Kargar St., Tehran, \\ 1439955941, Iran \\ †Corresponding Author Email: m.tahani@ut.ac.ir
}

(Received October 20, 2015; accepted December 10, 2015)

\begin{abstract}
In this study aerodynamics analysis of full scaled Vestas V47 wind turbine is carried out by the use of modified blade element momentum (BEM) theory and computational fluid dynamics (CFD). In order to determine accurate results BEM theory is programmed by considering drag coefficient, Glauert correction and Prandtle tip loss factor. CFD simulation is determined employing $\mathrm{k}-\mathrm{w}$ sst turbulence model and periodic boundary condition. The investigation outcomes are compared with each other. To validate CFD and BEM results, the only available data is real field measurement that is done by Vestas Company and power is compared with these data. Finally, according to the accuracy of results and computational cost, it is obtained that BEM is more applicable in engineering estimations.
\end{abstract}

Keywords: Wind turbine; CFD; Aerodynamic; BEM.

\section{NOMENCLATURE}

$\begin{array}{ll}a_{c} & \text { critical axial induction factor } \\ a & \text { axial induction factor } \\ a^{\prime} & \text { tangential induction factor } \\ C_{m} & \text { moment coefficient } \\ C_{N} & \text { normal force coefficient } \\ C_{L} & \text { lift coefficient } \\ C_{D} & \text { drag coefficient } \\ F & \text { Prandtle correction factor } \\ r & \text { radius } \\ T & \text { torque }\end{array}$

\section{INTRODUCTION}

Renewable energy studies have been growing rapidly due to rising energy demand, finite fossil fuels and environmental concerns. Wind energy is one of the fastest growing forms of clean energy resources. Wind turbines play significant role in providing economic power to satisfy today's huge demand of sustainable and clean forms of energy. Nowadays, a major concern of the wind turbine designers is their power efficiency and overall performance.

The most popular methods to predict aerodynamic behaviour of wind turbine are BEM, CFD, vortex method, Dynamic stall model and experiment. Among these methods experimental analysis is

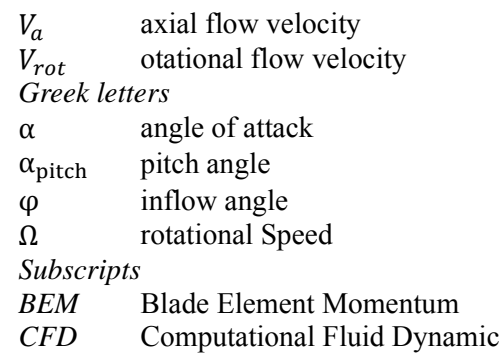

more expensive and time consuming. These experiments are so expensive because of wind turbine size and required tunnel size. Therefore the experimental data are limited in few numbers of laboratories such as NASA Ames $24.4 \mathrm{~m} \times 36.6 \mathrm{~m}$ wind tunnel and DNW.

Due to the importance of aerodynamic behaviour of wind turbine to investigate performance and structural behaviour of the turbine, mathematical and numerical models have become significant. In the vortex method effects of wake rotation in downstream are considered by replacement of the real flow through the rotor by an inviscid fluid flow through an equivalent vortex system (Abedi, 2011). Due to the time dependency nature of flow around the wind turbine blade and operation of the blade near stall angle, dynamic stall models can be used 
for aerodynamic analusis. Dynamic stall models are empirical and semiemperical which are developed recently. Hansen et al. (2004) developed a BeddoesLeishman model by ignoring the effect of compressibility and leading edge separation.

Computational fluid dynamic (CFD) is based on Navier-Stocks equations has potential to provide realistic simulation of the turbine flow field and can be used to solve the complex flow over the wind turbine. Yelmule and Anjuri (2013) performed CFD simulation of NREL Phase VI rotor with RANS sst k-omega model by means of Ansys CFX software and validate and verified their result with experimental and other aerodynamic method. Wang et al. (2012) studied blade-tower interaction of NREL Phase VI wind turbine in upwind configuration by using the open source OpenFOAM tools coupled with arbitrary mesh interface (AMI) method. Hsu et al. (2014) presented aaerodynamic validation study of Phase VI wind turbine by performing finite element arbitrary LagrangianEulerian-variational. Lanzafame et al. (2013) introduced the development of CFD analysis of a wind turbine. Their strategy was on generating a high quality mesh and optimizing turbulence models and optimized the spatial discretization, the mesh and the SST correlation parameters.

BEM method presented by Glauert (1948) enables to calculate the steady loads and power. This method with some modifications often predicts the performance of wind turbines with acceptable accuracy and also it has cheap computational run time. Yang et al. (2014) presented application of BEM theory without corrections on a wind turbine. But, airfoil data including lift and drag coefficients are determined from 3D CFD analysis of the wind turbine which are loaded in the BEM code. Good agreement was obtained when compared with the experimental data. Vazet al. (2011) presented a mathematical method based on BEM theory for horizontal axis wind turbine which took in to account the effect of wake on rotor plane. Lanzafame and Messina (2010) investigate performance of horizontal axis wind turbine in continuously operating at its maximum power coefficient based on BEM code. Because of low computational cost and accurate results, BEM is employed in most aerodynamic and aeroelastic analysis of wind turbine blade.

Herein aerodynamic analysis of an industrial scaled wind turbine is implemented using BEM and CFD. Most of aerodynamics analysis of wind turbine is implemented on simple wind turbine with short length model of wind turbine and only one type of airfoil, but Vestas V47 geometry is more complex and has tapered and twist along blade length in addition to, this turbine is designed with different airfoils. To validate the output result, the output power is compared with real field measurements.

\section{Wind TURBINE BLADE SPECIFICATION}

In this study, the V47-660 $\mathrm{kW}$ wind turbine blade is selected as a case study and required information of this turbine is presented in Table 1.

Table 1 V47-660kW wind turbine information

\begin{tabular}{|c|c|c|c|}
\hline \multicolumn{2}{|c|}{ Operational Data } & \multicolumn{2}{c|}{ Rotor } \\
\hline $\begin{array}{c}\text { Cut-in wind } \\
\text { speed }\end{array}$ & $4 \mathrm{~m} / \mathrm{s}$ & Diameter & $47 \mathrm{~m}$ \\
\hline $\begin{array}{c}\text { Rated wind } \\
\text { speed }\end{array}$ & $15 \mathrm{~m} / \mathrm{s}$ & Swept area & $\begin{array}{c}1735 \\
\mathrm{~m}^{2}\end{array}$ \\
\hline $\begin{array}{c}\text { Cut-out } \\
\text { wind speed }\end{array}$ & $25 \mathrm{~m} / \mathrm{s}$ & $\begin{array}{c}\text { Number of } \\
\text { blades }\end{array}$ & 3 \\
\hline $\begin{array}{c}\text { Rotational } \\
\text { speed }\end{array}$ & $\begin{array}{c}28.5 \\
\mathrm{rpm}\end{array}$ & $\begin{array}{c}\text { Maximum } \\
\text { chord }\end{array}$ & $\begin{array}{c}2087 \\
\mathrm{~mm}\end{array}$ \\
\hline $\begin{array}{c}\text { Rated } \\
\text { power }\end{array}$ & $660 \mathrm{~kW}$ & $\begin{array}{c}\text { Minimum } \\
\text { chord }\end{array}$ & $\begin{array}{c}282.5 \\
\mathrm{~mm}\end{array}$ \\
\hline
\end{tabular}

The geometry of V47-660 kW wind turbine blade includes five different airfoils which are FFA-W3, MIX, NACA 63-600, NACA 63-450 and NACA 63150. These five types of airfoil are presented in Fig. 1.

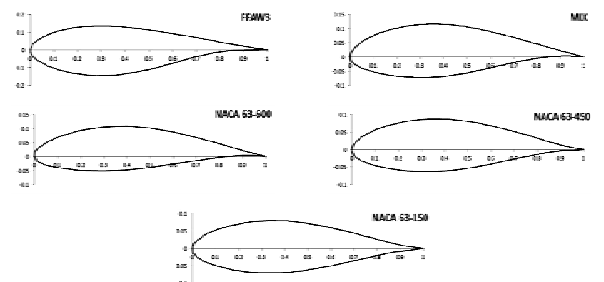

Fig. 1. Airfoil cross section of the blade.

Because of aerodynamical and structural considerations, the blade has twists and tapers from its root to tip. The cross sectional models of the blade depicting tapered and twisted blade are shown in Fig. 2.

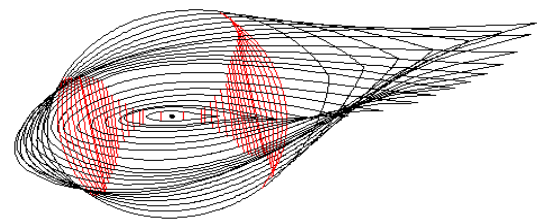

(a)

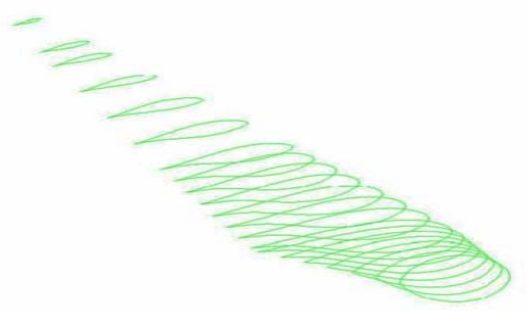

(b)

Fig. 2. (a) Twisted and (b) tapered cross sections along the blade length.

\section{Aerodynamic Analysis}

\subsection{BLADE ELEMENT MOMENTUM (BEM)}

BEM combines the Blade Element and one 
dimensional Momentum theory. In this method the wind turbine blades are divided into a certain numbers of independent elements along the length of blade. At each section, 2-D force equilibrium is developed considering produced torque and trust. At the same time momentum balance for annular element is applied. Finally a set of equations derived that can be solved for each blade section. This method is extensively explained in reference (Hansen 2008). The airfoil characteristics including lift and drag coefficients using in BEM code are determined by means of XFOIL in which viscousinviscid analysis methods have been integrated (Drela 2001).

Because of the rotational flow, the angle of attack at each section of blade is determined as below:

$$
\alpha=\alpha_{\text {pitch }}-\varphi
$$

Where $\alpha_{\text {pitch }}$ is pitch angle and $\varphi$ is inflow angle of attack calculated as it follows below:

$\varphi=\arctan \frac{\mathrm{V}_{\mathrm{a}}}{\mathrm{V}_{\text {rot }}}$

$\mathrm{V}_{\mathrm{a}}$ and $\mathrm{V}_{\text {rot }}$ are induced velocities in the rotor plane that are determined by means of axial and tangential induction factors as following:

$V_{\mathrm{a}}=(1-\mathrm{a}) \mathrm{V}_{0}$

$\mathrm{V}_{\text {rot }}=\left(1+\mathrm{a}^{\prime}\right) \Omega \mathrm{r}$

The normal and tangential force coefficients are determined by projecting lift and drag coefficients in corresponding directions.

$C_{\mathrm{N}}=\mathrm{C}_{\mathrm{L}} \cos (\varphi)+\mathrm{C}_{\mathrm{D}} \sin (\varphi)$
$\mathrm{C}_{\mathrm{T}}=\mathrm{C}_{\mathrm{L}} \sin (\varphi)-\mathrm{C}_{\mathrm{D}} \cos (\varphi)$

Finally axial and tangential induction factors are determined as:

$\mathrm{a}=\frac{1}{\frac{4 \mathrm{~F} \sin ^{2} \varphi}{\sigma \mathrm{C}_{\mathrm{N}}}+1}$

$\mathrm{a}^{\prime}=\frac{1}{\frac{4 \mathrm{~F} \sin \varphi \cos \varphi}{\sigma \mathrm{C}_{\mathrm{T}}}-1}$

Where $\sigma$ is solidity and defined as the fraction of the annular area in the control volume is covered by the blade. Prandtl presented a correction factor F to axial and tangential induction factor to correct the assumption of an infinite number of blades (Hansen 2008):

$\mathrm{F}=\frac{2}{\pi} \cos ^{-1} \mathrm{e}^{-\mathrm{f}}$

And
$f=\frac{B(R-r)}{2 r \sin \varphi}$

Moreover, Glauert correction is considered when the axial induction factor becomes large and momentum theory breaks down. The critical value for $a$ is equal to 0.2 and for $a>a_{c}$ the following equation is used:

$=\frac{1}{2}\left[2+k\left(1-2 a_{c}\right)\right.$

$\left.-\sqrt{\left(k\left(1-2 a_{c}\right)+2\right)^{2}+4\left(k a_{c}^{2}-1\right)}\right]$

Where:

$k=\frac{4 \mathrm{~F} \sin ^{2} \varphi}{\sigma \mathrm{C}_{\mathrm{N}}}$

The following algorithm is applied independently to each section for calculating aerodynamic loadings:

1. Initial guess for a and $\mathrm{a}^{\prime}$

2. Calculating inflow angle (Eq. 2)

3. Calculating local angle of attack (Eq. 1)

4. Reading lift and drag coefficient from XFOIL

5. Calculating axial and tangential induction factor (Eqs. 7 and 8)

6. If "a" is higher than critical value, update it using Eq. 15.

7. If estimated error of "a" and "a'"are more than considered tolerance, go to step 2 else go to next step

8. Calculate aerodynamic forces

Fig. 3 shows aerodynamics coefficients distribution along blade length. Due to the higher angle of attack and thicker airfoils close to the blade root than the other locations, drag coefficient is high and lift coefficient is low.
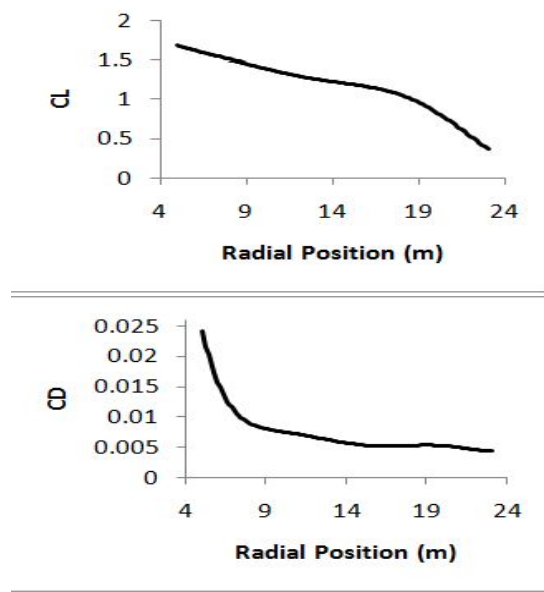

Fig. 3. Distribution of aerodynamics coefficients along blade length.

Fig. 4 shows comparison between output power was determined by the use of BEM and real field measurement data. An important condition for wind turbine is around rated speed. Real field 
measurement reported $14 \mathrm{~m} / \mathrm{s}$ for rated speed that output power reached $660 \mathrm{kw}$ (rated power). Also BEM determined rated speed equal to the $13 \mathrm{~m} / \mathrm{s}$ that this error is acceptable.

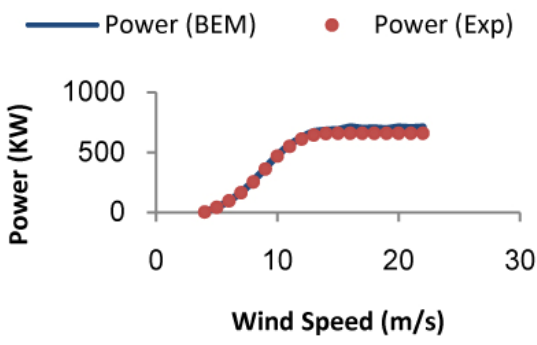

Fig. 4. Comparing BEM output power with control system with real field measurement data

In Fig. 5 the power coefficient was determined by BEM is validated with real field measurement data. Power coefficient shows that converting the wind energy to the electrical power how efficient wind turbine is. As shown in Fig. 5, real field measurement data reported the efficiency of the turbine is largest at wind speed around $7.5 \mathrm{~m} / \mathrm{s}$ equal to the 0.469. Also, BEM determined the highest power coefficient at wind speed around 8 $\mathrm{m} / \mathrm{s}$, that equals to the 0.498 .

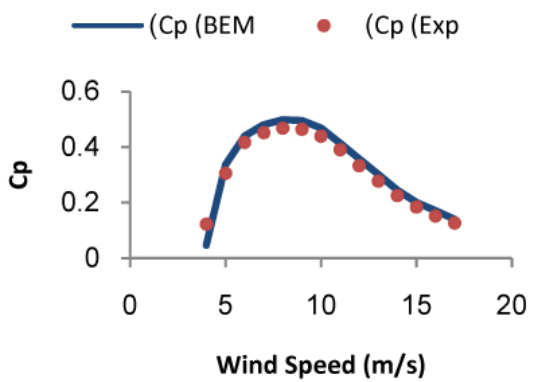

Fig. 5. Comparing BEM output power coefficient with control system with real field measurement.

\subsection{COMPUTATIONAL FLUID DYNAMICS (CFD)}

The CFD simulation of the blade is accomplished by considering one blade with periodic boundary conditions. This will be led to a considerable reduction in simulation. Since the free stream velocity is known, boundary condition at inlet is taken as velocity-inlet normal to the turbine. On the other hand, despite the inlet, pressure is known for outlet instead of velocity, which is the atmosphere condition. As a result, gauge pressure is set for outlet. Fig. 6 shows the generated mesh around the blade. Generally it has two different types of mesh which are rotary and stationary parts and near the blade surface boundary layer mesh is utilized. The computations have been done with 4,500,000 elements. The far filed distance in domain is chosen great enough (15 times characteristic length which is blade length), so that the simulation gives reliable results.

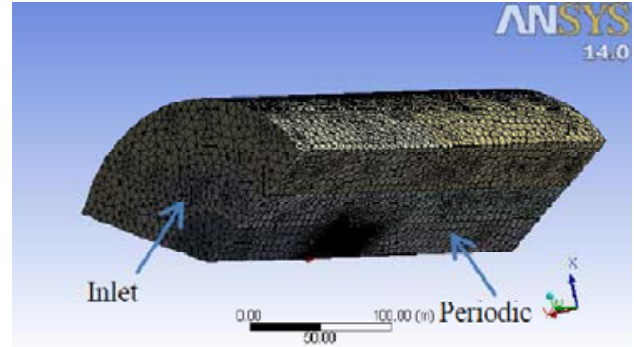

(a)

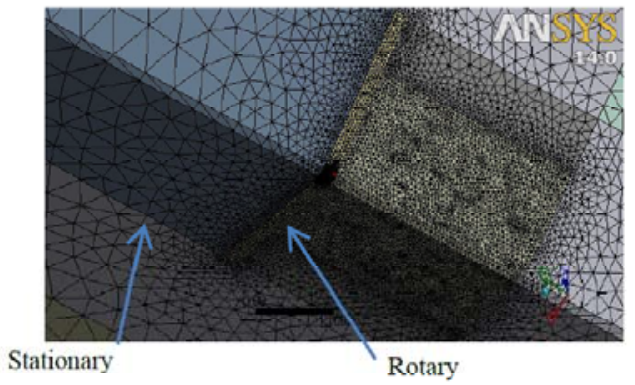

(b)

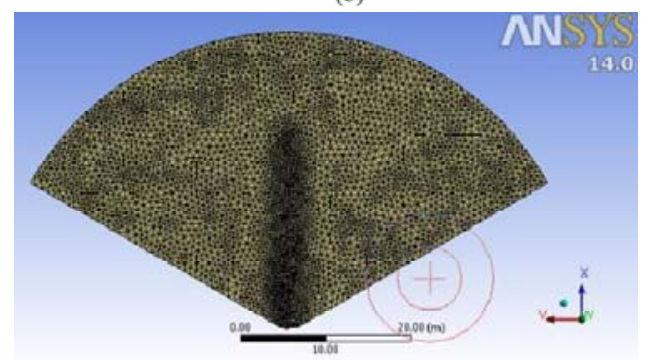

(c)

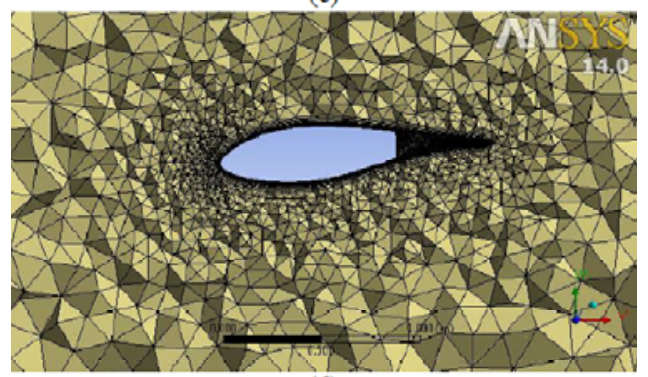

(d)

Fig. 6. Generated mesh around wind turbine blade.

Flow separation is a phenomena which is critical in wind turbine (Lanzafame 2013). So, k-omega Shear Stress Transport (SST) is applied as turbulence model. In this model a differential equation for specific rate of dissipation $(\omega)$ is solved except for dissipation rate of turbulent energy $(\varepsilon)$. Menter introduced SST model for $\mathrm{k}-\omega$ to integrate exact and strong $\mathrm{k}-\omega$ equations near the wall region, with independent $\mathrm{k}$ - equations in the far field (Menter 1994). According to the boundary layer mesh on the blade, $y^{+}$number is about 5 along blade length.

To calculate the output power of the turbine, moment coefficient value should be used. Having angular velocity and moment about rotating axis of turbine power delivered by turbine can be defined. 
The torque $\mathrm{T}$ can be calculated by the following equation:

$T=\frac{1}{2} \rho \cdot A_{S} \cdot U_{\infty}^{2} \cdot R \cdot C_{m}$

where $\rho$ and $U_{\infty}$ are density and velocity of free stream, respectively, $C_{m}$ (moment coefficient) is obtained directly through developed CFD code, $A_{S}$ is circle area swept by rotating blades and $\mathrm{R}$ is blade radius in SI units. Assuming standard condition we use the value of $1.225 \mathrm{~kg} \cdot \mathrm{m}^{-3}$ for air density.

A comparison between obtained results of BEM, CFD and experiment are presented in Table 2.

Table 2 Verification of BEM by CFD

\begin{tabular}{|c|c|c|c|}
\hline \multirow{2}{*}{$\begin{array}{c}\text { Wind } \\
\text { Speed } \\
(\mathrm{m} / \mathrm{s})\end{array}$} & \multicolumn{3}{|c|}{ Power $(\mathrm{kW})$} \\
\cline { 2 - 4 } & BEM & CFD & Exp. \\
\hline 7 & 168 & 150 & 166 \\
\hline 10 & 477 & 400 & 450 \\
\hline 14 & 684 & 580 & 651 \\
\hline 17 & 701 & 630 & 660 \\
\hline
\end{tabular}

\section{CONCLUSION}

The main objective of this study was aerodynamic analysis of a complex geometry wind turbine using two methods which are BEM and CFD. BEM theory, which is a well-known theory for aerodynamic analysis of a wind turbine, is implemented by considering two corrections and drag coefficients. Also CFD simulation was done to compare with BEM results. In the CFD analysis periodic boundary condition is used and k- $\omega$ SST turbulence model was chosen. As a result, it is observed that Compare to the real field measurement BEM result could predict output power with low computational cost. So, Du to the mentioned advantages of BEM, this theory is more willing to use to obtain aerodynamic loads for structural analysis such as aero elastic analysis.

\section{REFERENCES}

Abedi, H. (2011) Aerodynamic Load on Rotor Blades, Master Thesis, Chalmers University of Technology, Sweden.
Drela, M. (2001). XFOIL 6.94 User Guide, MIT Aero and Astro Harold Youngren, Aerocraft, Inc. Glauert H. (1948). The Elements of Aerofoil and Airscrew Theory. Cambridge University Press.

Hansen M. O. L. (2008) Aerodynamics of Wind Tur,bine, second edition, Earthscan Publication.

Hansen, M. H., Gaunaa, M., and Madsen, H. Aa., A Beddoes-Leishman type dynamic stall model in state-space and indicial formulation, Risø-r1354(en), Risø National Laboratory, Denmark.

Hsu, M. C., I. Akkerman, and Y. Bazilevs (2014) Finite element simulation of wind turbine aerodynamics: validation study using NREL Phase VI experiment, Wind Energ, 17, 461-481.

Lanzafame, R., S. S. Mauro, and M. Messina (2013).Wind turbine CFD modeling using a correlation-based transitional model, Renewable Energy 52, pp.31-39.

Lanzafame, R. and M. Messina. (2010). Horizontal axis wind turbine working at maximum power coefficient continuously, Renewable Energy 35, 301-306

Menter, F. R. (1994). Two-Equation Eddy-Viscosity Turbulence Models for Engineering Applications. AIAA Journal, 32(8), 1598-1605.

Vaz, J. R. P., J. T. Pinho, and A. L. A. Mesquita (2011) An extension of BEM method applied to horizontal-axis wind turbine design. Journal of Renewable Energy, 36, 1734-1740.

Wang, Q., H. Zhou, and D. Wan (2012) Numerical Simulation of Wind Turbine Blade-Tower Interaction. J. Marine Sci. Appl. 11. pp. 321327.

Yang, H, W. Shen, H. Xu, Z. Hong, and C. Liu (2014) Prediction of the wind turbine performance by using BEM with airfoil data extracted from CFD, Renewable Energy, 1-9.

Yelmula M. M. and E. Anjuri (2013). CFD predections of NREL Phase VI rotor experiments in NASA/AMES wind tunnel, International Journal of Renewable Energy Research, 3(2) 